\title{
Transhepatic Access to the Atrioventricular Ring for Delivery of Radiofrequency Energy
}

\author{
PETER FISCHBACH, M.D., ROBERT M. CAMPBELL, M.D.,* \\ EDWARD HULSE, M.D.* RALPH MOSCA, M.D., BRIAN ARMSTRONG, \\ THOMAS R. LLOYD, M.D., and MACDONALD DICK II, M.D.
}

From the Michigan Congenital Heart Center, C.S. Mott Children's Hospital, Departments of Pediatrics and Surgery, University of Michigan, Ann Arbor, Michigan; and the *The Children's Heart Center, Egleston Children's Hospital, Atlanta, Georgia

\begin{abstract}
Transhepatic Ablation. Introduction: Transcatheter radiofrequency ablation is an effective, safe treatment of arrhythmias in children. However, despite technical advances, patients with obstructed venous access to the heart have not been candidates for this treatment.

Methods and Results: Two children $(8.2$ and $10.9 \mathrm{~kg})$ with complex congenital heart disease, supraventricular tachycardia, and obstructed venous access underwent successful radiofrequency ablation of either a right (one patient) or left (one patient) accessory pathway using the transhepatic route to the heart.

Conclusion: This experience underscores the usefulness of the transhepatic approach in small patients, in patients with limited venous access, and in patients with complex congenital heart disease. (J Cardiovasc Electrophysiol, Vol. 8, pp. 512-516, May 1997)
\end{abstract}

arrhythmia, cardiac catheterization, children, congenital heart disease, radiofrequency ablation, supraventricular tachycardia, transhepatic catheterization

\section{Introduction}

Radiofrequency ablation (RFA) has proved to be an effective and safe treatment of many different arrhythmias in pediatric patients. The expanding use of special techniques such as transseptal entry to the left atrium, single catheters serving two functions, thinner caliber catheters, and long vascular sheaths has led to the successful application of radiofrequency current to complex arrhythmias occurring in children of all sizes with either structurally normal hearts or complex congenital heart disease. ${ }^{1-5}$ Despite these technical advances, patients with venous obstruction and thus absence of venous access to the heart may be denied RFA. Two forms of complex congenital heart defects especially subject to arrhythmias are Ebstein's anomaly of the tricuspid valve and

Address for correspondence: Macdonald Dick II, M.D., Room 1310, Box 0204, University of Michigan Medical Center, 1500 East Medical Center Dr., Ann Arbor, MI 48109-0204. Fax: 313936-9470.

Manuscript received 28 November 1996; Accepted for publication 22 January 1997. congenitally corrected transposition of the great arteries. Both of these cardiac malformations are associated with accessory pathways supporting AV reentry tachycardia. ${ }^{6-8}$ In this report, we present two patients with complex congenital heart disease, supraventricular tachycardia (SVT), and obstructed vascular access to the heart who were successfully treated for SVT by RFA of their accessory pathway using transhepatic cardiac catheterization. $^{9}$

\section{Patient 1}

A 26-month-old girl (10.9 kg) with situs solitus, dextrocardia, congenitally corrected transposition of the great arteries, a ventricular septal defect (VSD), pulmonary atresia, a normal leftsided tricuspid valve, and recurrent SVT received a Blalock-Taussig shunt on the 19th day of life. Digoxin and propranolol were administered to control her SVT, with only partial success. Electrophysiologic study, limited by known bilateral femoral venous occlusion, was completed through the right subclavian vein. This study demonstrated 
a concealed posterior septal accessory pathway supporting her SVT. However, because neither the left atrium nor the coronary sinus could be entered, the exact location of the accessory pathway could not be determined. Surgical repair of the VSD and placement of a left ventricular to pulmonary artery conduit was performed, but planned mapping and ablation of the accessory pathway was not attempted because access to the AV ring was limited by the dextrocardia and the conduit. Early after surgery, the SVT (230 beats/min) recurred. On postoperative day 7 , an electrophysiologic study through surgically placed transthoracic epicardial atrial and ventricular leads was performed. The technique of transhepatic venous access to the heart has been described in detail previously. ${ }^{9}$ Briefly, a 22-gauge Chiba needle with stylet (Cook, Inc., Bloomington, IN, USA) was introduced percutaneously into the liver in the midaxillary line half way between the diaphragm and the lower margin of the liver. The needle was advanced under fluoroscopic guidance to the midclavicular line. The stylet was then removed, and the needle was withdrawn slowly as a small volume of iohexol was gently and continuously instilled. When the contrast filled a hepatic venous radical and flowed cephalad toward the right atrium, a 0.018-inch mandril guidewire (Cook, Inc.) was then advanced through the needle into the hepatic vein and the right atrium. A 4-French coaxial dilator was then advanced over the wire and positioned in the low right atrium. A 0.035 -inch J-wire was

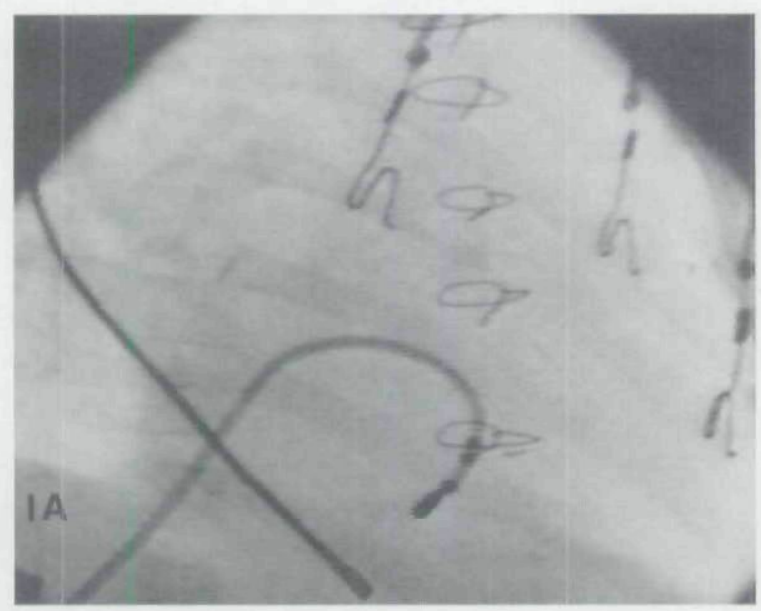

exchanged for the inner dilator, and the mandril wire and the outer dilator were removed. A 6French curved transhepatic sheath was then advanced over the 0.035 -inch $\mathrm{J}$-wire into the right atrium. A 6-French steerable quadripolar electrode catheter was then advanced through the sheath. Mapping demonstrated that the shortest right-sided retrograde activation time, measured during SVT when the catheter was positioned in the right posteroseptal area, was $81 \mathrm{msec}$. Application of radiofrequency current at this site was unsuccessful. Access to the left AV ring was accomplished by exchanging the transhepatic 6-French sheath for a 6-French pediatric transseptal sheath, which was advanced to the left atrium (Fig. 1). Mapping of retrograde left atrial activation during SVT revealed that the shortest VA interval was $60 \mathrm{msec}$ at the left posteroseptal ring. RFA at this site terminated SVT 2.5 seconds after initiation of the fifth application (Fig. 2). Postablation electrophysiologic study failed to induce SVT. The patient was discharged home the next day; she has remained free of SVT during the 3 months of follow-up.

\section{Patient 2}

A 20-month-old boy $(8.2 \mathrm{~kg})$ with complex Ebstein's anomaly including pulmonary atresia, preexcitation, and SVT had received a bidirectional Glenn shunt at 6 months of age. His SVT had been partially controlled with amiodarone. Due to the Glenn shunt and the known bilateral femoral

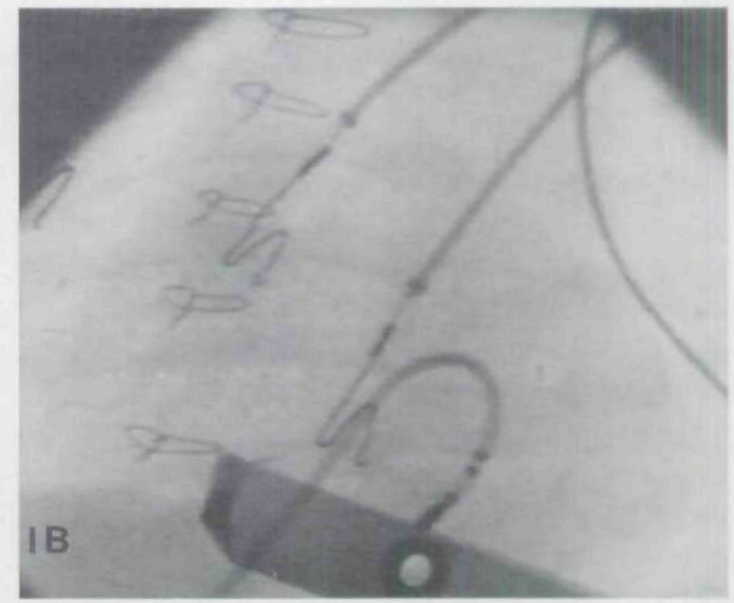

Figure 1. Radiographs from patient 1 displaying the transhepatic, transseptal sheath deployed through the liver, into the right atrium, and across the atrial septum into the left atrium. Note the diagonal course directed from the patient's right side rather than from her leg. The steerable electrode catheter is passed through the sheath so that the oversized electrode tip (4 mm) is positioned at the left posterior AV ring. Note that the right-sided morphologic left ventricle is directed to the right and posteriorly toward the vertebral bodies. (A) Right anterior oblique projection; (B) left anterior oblique projection. 


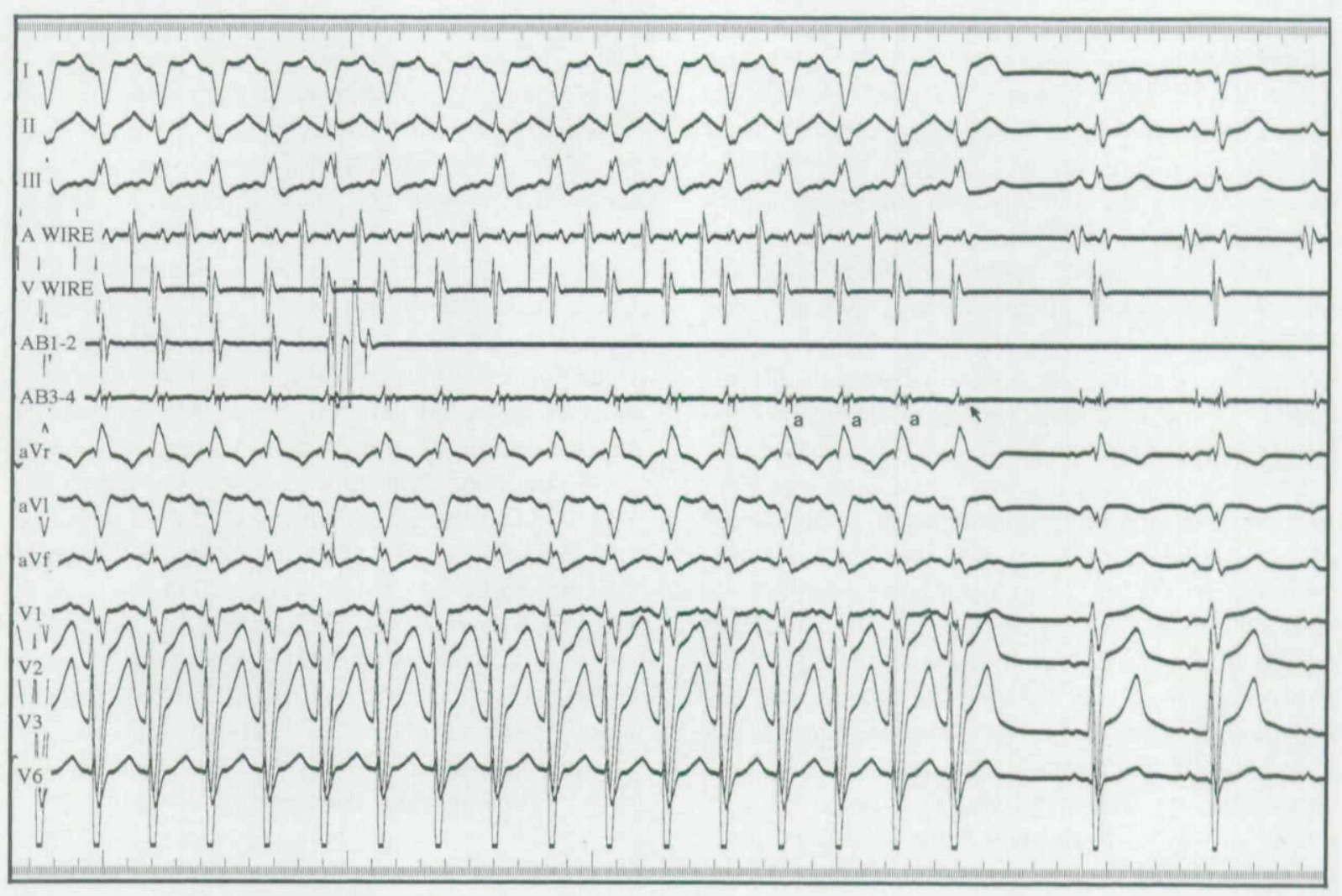

Figure 2. ECG and electrophysiologic tracings during delivery of radiofrequency current in patient 1. Note the absence of a retrograde " $a$ " wave (arrow, recorded through electrodes 3 and 4 [AB3-4] on the mapping/ablating catheter) 2.5 seconds after initiation of radiofrequency current terminating the tachycardia. Paper speed $=50 \mathrm{~mm} / \mathrm{sec} . \quad a=$ atrial electrogram; AB1-2 $=$ electrodes $1-2$ on the mapping/ablation catheter; $A B 3-4=$ electrodes $3-4$ on the mapping/ablation catheter; $A$ WIRE $=$ bipolar atrial electrogram recorded through transthoracic epicardial atrial wires; $V$ WIRE = bipolar ventricular electrogram recorded through transthoracic epicardial ventricular wires.

vein occlusion following prior cardiac catheterizations, all standard access to the right atrium was blocked. Therefore, using the transhepatic approach similar to that described for patient 1, a 7-French sheath was advanced into the right atrium. A transesophageal 4-French bipolar electrode catheter was used for atrial recording and pacing. Mapping of the right-sided AV ring was performed anterogradely during preexcited sinus rhythm and retrogradely during SVT. With the steerable quadripolar electrode catheter passing through the hepatic veins into the right atrium toward the atrial septum, a $135^{\circ}$ anterior deflection was delivered to the steerable tip. Traction was then applied to the catheter and sheath as a unit, bringing the electrode tip firmly in contact with the right $\mathrm{AV}$ groove (Fig. 3). Flexion and extension of the catheter tip moved the catheter tip along the lateral AV groove, whereas counterclockwise and clockwise rotation of the catheter and curved sheath as a unit moved the tip into the atrium or ventricle, respectively. Using this technique, the patient was found to have a right lateral accessory pathway. Radiofrequency energy delivered during the seventh application at the site with maximal local preexcitation during sinus rhythm and the shortest retrograde conduction time during $\mathrm{SVT}^{10}$ along the right $\mathrm{AV}$ groove terminated both the preexcitation and SVT. Recurrence of the SVT and preexcitation 10 minutes later required an additional four applications to achieve permanent ablation of the accessory pathway. The patient was discharged home the next day; he has remained free of preexcitation and SVT at 3 months of follow-up.

\section{Discussion}

Application of RFA requires the operator to maneuver specifically designed electrode catheters to the potential ablation site. This report is the first 

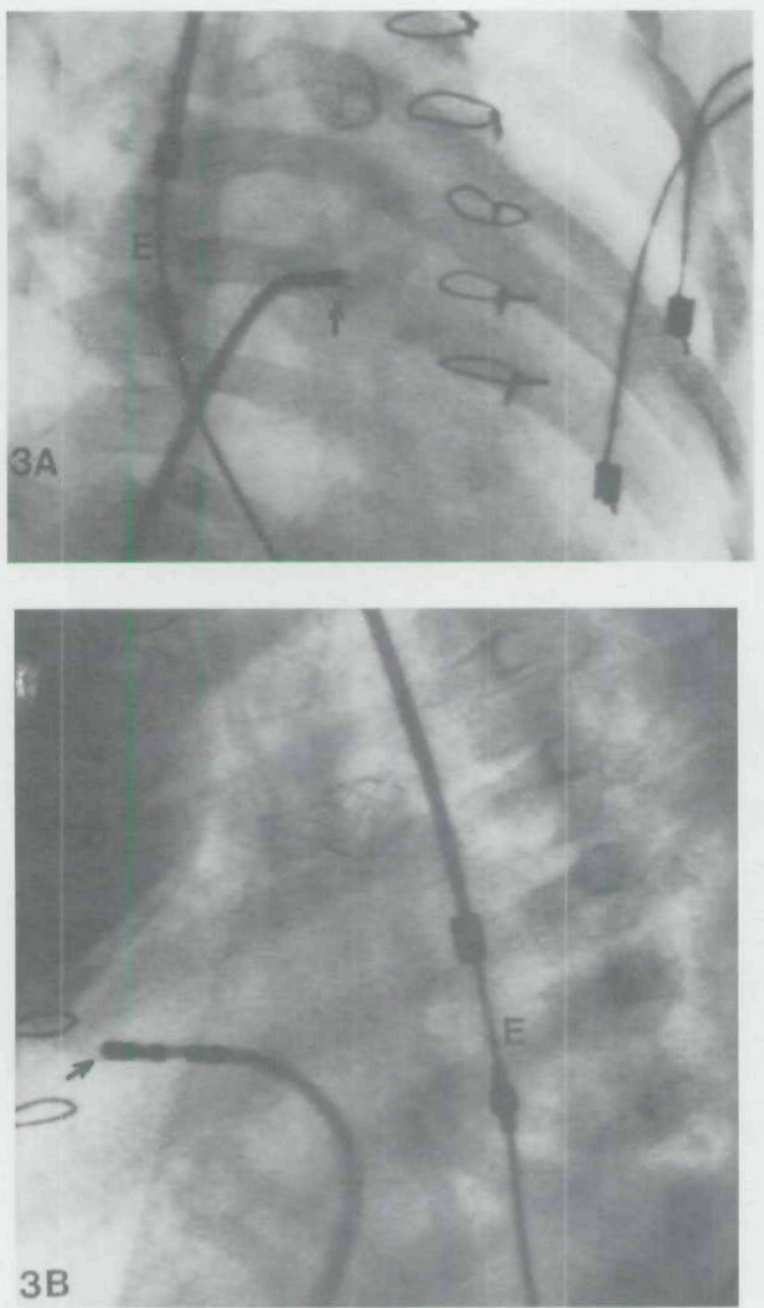

Figure 3. Radiographs from patient 2 displaying the transhepatic sheath and steerable electrode catheter deployed through the liver into the right atrium and then flexed and rotated anteriorly and to the right so that the oversized electrode tip ( $4 \mathrm{~mm}$, arrow) abuts the right lateral free wall along the AV groove. The bipolar electrode transesophageal catheter $(E)$ can be seen. There is a stent in the left pulmonary artery. (A) Right anterior oblique projection; (B) left anterior oblique projection.

to describe RFA using the transhepatic catheterization approach and highlights the difficulties that may be encountered when performing RFA in small patients with complex congenital heart disease and limited venous access. Even among patients with normal structural hearts and right free-wall pathways, a stable catheter position against the right AV groove is often difficult to achieve, and ablation at this site is less successful. ${ }^{3}$ Prior cardiac catheterization may result in vein or artery occlusion or injury.9.11.12 Surgical procedures such as the Glenn, Mustard, Senning, and modified Fontan (lateral tunnel) operations, as well as deep vein thrombotic disease in the lower extremities and pelvis, may exclude portions of the right atrium, which is often the site of the RFA target.

Recently, Shim and associates ${ }^{9}$ reported a series of infants and children with limited vascular access who underwent diagnostic and interventional catheterizations using the transhepatic approach and 5- to 8-French sheaths with no significant adverse effects. Interventions in this series included balloon valvuloplasty and angioplasty and device closure of an atrial septal defect. Access to the right atrium was quickly, reliably, and safely achieved in this cohort of patients with no other venous access.

The experience with these two patients demonstrates several advantages of transhepatic cardiac catheterization for RFA. The first is that the technique allows the introduction of electrode catheters to both the right and left sides of the heart as well as to the region of the AV ring on both sides when standard access is unavailable. Retrograde arterial access to the left heart with large-gauge catheters has not proved to be an attractive technique in small children. In a report of children undergoing aortic valvuloplasty using the retrograde approach from the femoral artery(ies), $12 \%$ of all patients developed femoral arterial thrombi or damage noted at the time of the valvuloplasty; in patients $<1$ year of age, $32 \%$ developed femoral arterial thrombi or damage. ${ }^{11}$ This same group noted femoral artery injury in 3 of 5 patients $<12$ months of age at aortic valvuloplasty at intermediate follow-up. ${ }^{12}$ In addition, Doppler-detected aortic regurgitation has been noted in children who underwent ablation of left-sided pathways using the retrograde route to the left $\mathrm{AV}$ ring. ${ }^{13}$ Finally, similar success for ablation of left-sided accessory pathways using either the transseptal or retrograde approaches has been reported. ${ }^{14}$ Thus, this combined transhepatic transseptal technique not only preserves a good outcome, but also avoids passing a large-caliber ablation catheter through the femoral artery placing both the artery and aortic valve at risk..$^{9,11-14}$

The second patient also demonstrated an unexpected, but important, benefit of the transhepatic catheter procedure. The course of the catheter, as it passed through the hepatic vein and the inferior vena cava into the right atrium toward the atrial septum and was deflected and curved anteriorly, afforded an excellent angle of attack to the right lateral $\mathrm{AV}$ ring providing an optimal position for RF energy delivery at that site. Furthermore, the 
shorter distance between target site at the right AV ring and the entry point in the liver, compared to that in the groin, may provide greater torque control of the catheter tip. The Ebstein's anomaly or a dilated right atrium often confound efforts to achieve a stable ablation catheter position at the right lateral $\mathrm{AV}$ ring.

In conclusion, this experience demonstrates that transhepatic catheterization in the hands of an experienced operator is a rapid and safe route to both the right and left AV ring for delivery of radiofrequency energy. Furthermore, this experience underscores the usefulness of the technique in small patients, in patients with limited venous access, and in patients with complex congenital heart disease. The technique may be of special value not only when access is limited but also when precise, stable catheter placement along the right lateral AV groove is required.

\section{References}

1. Dick M, O'Connor B, Serwer GA, et al: Use of radiofrequency current to ablate accessory connections in children. Circulation 1991;84:2318-2324.

2. Van Hare GF, Lesh MD, Scheinman M, et al: Percutaneous radiofrequency catheter ablation for supraventricular arrhythmias in children. J Am Coll Cardiol 1991;17:1613-1620.

3. Kugler JD, Danford DA, Deal BJ, et al: Radiofrequency catheter ablation for tachyarrhythmias in children and adolescents. N Engl J Med 1994;330:1481-1488.

4. Saul JP, Hulse JE, Wang DE, et al: Catheter ablation of accessory atrioventricular pathways in young patients: Use of long vascular sheaths, the transseptal approach, and a retrograde left posterior parallel approach. J Am Coll Cardiol 1993;21:571-583.

5. Dick M II, Law IH, Dorostkar PC, et al: Use of the His/RVA catheter in children. J Electrocardiol 1996; 29(Suppl):14-20.

6. Schiebler GL, Adams P Jr, Anderson RC, et al: Clinical study of 23 cases of Ebstein's anomaly of the tricuspid valve. Circulation 1959;19:165-187.

7. Van Lingen B, Bauersfeld SR: The electrocardiogram in Ebstein's anomaly of the tricuspid valve. Am Heart J 1955;50:13-23.

8. Bharati S, Rosen K, Steinfeld L, et al: The anatomic substrate for pre-excitation in corrected transposition. Circulation 1980;62:831-842.

9. Shim D, Lloyd TR, Cho KJ, et al: Transhepatic cardiac catheterization in children: Evaluation of efficacy and safety. Circulation 1995;92:1526-1530.

10. Schwartz SM, Dick M II, Dorostkar PC, et al: Electrogram patterns associated with successful radiofrequency ablation of accessory pathways in children. Pediatr Cardiol 1996;17:137-142.

11. Rocchini AP, Beekman RH, Ben Shachar G, et al: Balloon aortic valvuloplasty: Results of the Valvuloplasty and Angioplasty of Congenital Anomalies Registry. Am J Cardiol 1990;65:784-789.

12. O'Connor BK, Beekman RH, Rocchini AP, et al: Intermediate-term effectiveness of balloon valvuloplasty for congenital aortic stenosis: A prospective follow-up study. Circulation 1991;84:732-738.

13. Minich LA, Snider AR, Dick M II: Doppler detection of valvular regurgitation after radiofrequency ablation of accessory connections. Am J Cardiol 1992; 70:116-117.

14. Dick M, Serwer GS, Armstrong B, et al: Access to leftsided accessory pathways in young patients: Transseptal vs retrograde aortic route. Circulation 1996; 94(Suppl I):I-120. 
This document is a scanned copy of a printed document. No warranty is given about the accuracy of the copy. Users should refer to the original published version of the material. 